\title{
Índice de salud de las lagunas costeras de Cayos Miskitos
}

\author{
Health index of the coastal lagoons from Miskitos Cays
}

\author{
Donald Williams Smith ${ }^{1}$ \\ Marcos Williamson ${ }^{2}$ \\ Norving Torres ${ }^{3}$ \\ Osmar Arróliga ${ }^{4}$ \\ José Mendoza ${ }^{5}$
}

\section{Resumen}

Los ecosistemas marino costeros de la costa Caribe Norte de Nicaragua, conforman una red de humedales interdependientes de gran importancia ecológica. En estos humedales sobresale un sistema de lagunas naturales, donde se reproducen y se desarrollan una cantidad de organismos acuáticos, que son la base de la economía y la alimentación, de las comunidades indígenas miskitas que habitan en la zona costera. Estas lagunas reciben agua de afluentes continentales y del mar Caribe, por tanto, dada la importancia de este sistema lagunar, en esta investigación cuantitativa se desarrolló una evaluación ecológica en las lagunas Bihmuna, Pahra, Karata y Wouhta, para conocer su estado de salud, analizando tres indicadores principales: Contaminación del agua, hábitat y pesquería. Los resultados demuestran buena calidad del agua con baja concentración de elementos y sus formas que se presentan, buena cobertura y conservación de hábitat, abundancia y diversidad de especies pesqueras. De modo que estas lagunas presentan buena condición de salud y deben mantener el uso actual para conservar sus valores naturales y que los comunitarios puedan hacer uso de los recursos de manera sostenible en el tiempo. Las capacitaciones que la universidad URACAN realiza son esenciales para la conservación de los humedales en el área.

Palabras clave: Índice; laguna; salud lagunar; mangle.

\section{Abstract}

The coastal marine ecosystems of the North Caribbean Coast of Nicaragua, make up a network of interdependent wetlands of great ecological importance. In these wetlands a system of natural lagoons stands out, where a number of aquatic organisms reproduce and develop, these organisms are the basis of the economy and food of the Miskito indigenous communities that inhabit the coastal zone. These lagoons receive water from continental tributaries and the Caribbean Sea, therefore, given the importance of this lagoon system, in this quantitative investigation an ecological evaluation was developed in the Bihmuna, Pahra, Karata and Wouhta lagoons, to know their state of health, analyzing three main indicators: Water pollution, habitat and fisheries. The results demonstrated good water quality with low concentration of elements and their forms, good habitat cover and conservation, abundance and diversity of fishing species. So these gaps have a good health condition and must maintain the current use to conserve their natural values and that the community can make use of the resources in a sustainable manner over time. The training that URACAN University carries out is essential for the conservation of wetlands in the area.

\footnotetext{
Licenciado en biología marina, Técnico de IREMADES. ORCID: http://orcid.org/0000-0002-1931-577X ; e- mail; ronaldw1981@yahoo.com

2 Msc. en Gobernanza y Desarrollo de Identidad, Director de IREMADES-CISA, URACCAN; e-mail: marcoswillni123@gmail.com ORCID: http://orcid.org/00000002-1887-1328

3 MSc. en Manejo de Vida Silvestre. Presidente FUNDAR.

4 Licenciado en Ecología. Técnico FUNDAR.

5 Licenciado en Biología. Técnico FUNDAR.
}

Recibido: 08/08/2019 - Aprobado: 30/08/2019 
Keywords: Index; lagoon; lagoon health; mangrove.

\section{Introducción}

El presente trabajo se realiza en el marco del proyecto: "Fortalecimiento de condiciones y capacidades de la población para la conservación y manejo sostenible de ecosistemas costeros de la Costa Caribe", financiado por la Comisión Europea, la Cooperación Austriaca para el desarrollo CAD-ADA y DKA Austria, y ejecutado por un consorcio conformado por la organización Austriaca de Cooperación para el desarrollo HORIZONT 3000, y la Universidad de las Regiones Autónomas de la Costa Caribe Nicaragüense (URACCAN).

Se realizó evaluación ecológica en la franja costera de la Reserva Biológica Cayos Miskitos, ubicada en la Región Autónoma de la Costa Caribe Norte de Nicaragua, para conocer el estado de salud del sistema lagunar. En el área predominan humedales costeros incluyendo lagunas naturales, áreas compactas de mangle y llanos temporalmente inundados. La reserva biológica Cayos Miskitos y su franja costera inmediata fue declarada Sitio RAMSAR por la UNESCO en el 2001, asignándole el número de referencia 1135.

Para conocer el estado de salud del sistema lagunar fueron evaluados tres indicadores principales: 1) Contaminación: se realizaron muestreos de agua para conocer a través de análisis físico químico la calidad del agua, con respecto a las concentraciones de los elementos y las formas en que se presentan. 2) Hábitat. En este indicador se consideraron los ecosistemas bosque de mangle, llanos inundados y turberas, identificando el área cubierta y las condiciones de conservación. 3) Pesquería. En este indicador se consideraron los muestreos en el área de las lagunas para conocer la biomasa presente de los organismos pesqueros.

Los análisis de los tres indicadores demuestran que este sistema lagunar, se encuentran en buen estado ambiental. Los parámetros físico químico del agua no ponen en riesgo la vida de los organismos acuáticos. Se debe continuar con la protección de estos ecosistemas como se ha venido realizando

\section{Revisión de literatura}

El Caribe nicaragüense es una zona privilegiada, producto de la interacción de una variedad de ecosistemas, entre ellos los humedales. Las altas precipitaciones y la existencia de tierras planas y bajas crean las condiciones para la presencia de ambientes acuáticos de gran tamaño como Cayos Miskitos, que forma parte de un corredor de humedales ricos en biodiversidad que parte en la península de Yucatán, sigue por Belice, Guatemala, Honduras, Nicaragua, Costa Rica y termina en Panamá. (Tabilo -. Valdivieso E., 1999).

Entre estos ambientes acuáticos se encuentran las lagunas costeras, consideradas depresiones someras (<10 m), con su eje principal paralelo a la costa, conectada al mar y separada de él por una barrera física (Kjerfve, 1994). Se ubican entre los ecosistemas de mayor productividad, además, son importantes tanto por su biodiversidad, como por las actividades socioeconómicas que sostienen (López, et al 2012).

Las lagunas costeras y estuarios representan el mejor índice de calidad de la cuenca. Así, el conocimiento de las principales variables fisicoquímicas ofrece claves invaluables para el manejo de las cuencas y ecosistemas costeros (Contreras y Castañeda, 2004).

Las lagunas costeras del Caribe Norte de Nicaragua, han sido poco estudiadas como consecuencia hay pocos registros de sus características fisicoquímicas. El presente estudio fue realizado en la estación seca, posiblemente en estación lluviosa los datos varíen, principalmente el oxígeno disuelto y la salinidad del agua, según Alzieu (1994), por sus características hidrológicas y ecológicas, las lagunas costeras muestran variación temporal de forma significativa. 
En general estas lagunas presentan buen estado de salud, según el análisis de los tres indicadores: contaminación, hábitat y pesquería, dado su estado natural y que no presentan descargas de aguas de áreas urbanas. Caso contrario se presenta en la Bahía Chesapeake, en Estados Unidos, donde según (Save de Bay (2016), sí reciben descargas de agua proveniente de industrias y aéreas urbanas.

\section{Materiales y métodos}

Para conocer el estado de salud del sistema lagunar de la zona costera de la reserva biológica Cayos Miskitos, se realizaron muestreos en cuatro lagunas (Bihmuna, Pahra, Karata y Wouhta). Se consideraron tres líneas de indicadores: Contaminación, hábitat y pesquería.

El grado de contaminación de las lagunas se obtuvo mediante la colecta de muestras de agua, las que fueron analizadas en el laboratorio del Centro de Investigación de Recursos Acuáticos (CIRA) de la Universidad Nacional de Nicaragua (UNAN), para conocer a través de análisis físico químico la calidad del agua, con respecto a las concentraciones de los elementos y las formas en que se presentan.

El estado del hábitat se obtuvo, analizando la cobertura y el grado de conservación de los ecosistemas: bosque de mangle, llanos inundados y turberas. En el manglar se delimitaron parcelas y se colectó información dasométrica de los árboles. En las áreas de llanos se realizaron transeptos para colectar información de la cobertura. Luego se realizó análisis SIG, para determinar a través de imágenes de satélite la cobertura de cada formación forestal.

Para conocer el estado del recurso pesca, se realizaron muestreos de captura. A los organismos capturados se les colectó el peso e información morfométrica, para determinar la biomasa de organismos pesqueros en cada laguna. Además, para conocer la población de cangrejo (Aratus pisoni), se realizaron parcelas de muestreo de $1 \mathrm{~m}^{2}$, para conocer la densidad de los individuos por área.

Los valores de la información colectada en cada una de las cuatro lagunas, sirvió para construir una tabla con los valores asignados según a la metodología de Save de Bay (2016). Se asignó una puntuación a cada indicador y luego fue promediado el puntaje en las tres categorías para determinar el estado general de cada laguna y conocer el estado de salud todo el sistema lagunar. Cuadro No. 1.

Cuadro No. 1. Puntuación y grado de los indicadores, de acuerdo al estado de conservación

\begin{tabular}{|l|l|l|l|}
\hline \multicolumn{1}{|c|}{ Puntuación } & \multicolumn{1}{c|}{ Grado } & \multicolumn{1}{c|}{ Puntuación } & \multicolumn{1}{c|}{ Grado } \\
\hline 70 o mas & A & $40-44$ & C \\
\hline $65-69$ & A- & $34-39$ & C- \\
\hline $60-64$ & B+ & $30-33$ & D+ \\
\hline $55-59$ & B & $25-29$ & D \\
\hline $50-54$ & B- & $20-24$ & D- \\
\hline $45-49$ & C+ & 190 menos & F \\
\hline
\end{tabular}

\section{Resultados y discusión}

De acuerdo al análisis obtenido de cada uno de los indicadores logramos determinar que en general las lagunas naturales presentan una buena condición de salud. Esto principalmente porque se mantiene las condiciones ambientales y naturales en los ecosistemas que las rodean. No existen fábricas ni industrias que aporten descargas de contaminantes químicos, ni grandes descargas de desechos domiciliares. El valor promedio calculado para el sistema lagunar fue de 44.10. Este valor es superior a lo reportado de la Bahía Chesapeake por Save de Bay (2016), quienes reportan un valor de 34(C-). Cuadro No. 2. 
Cuadro No. 2. Índice de salud de las lagunas naturales, valoración de los indicadores. Cayos Miskitos.2018

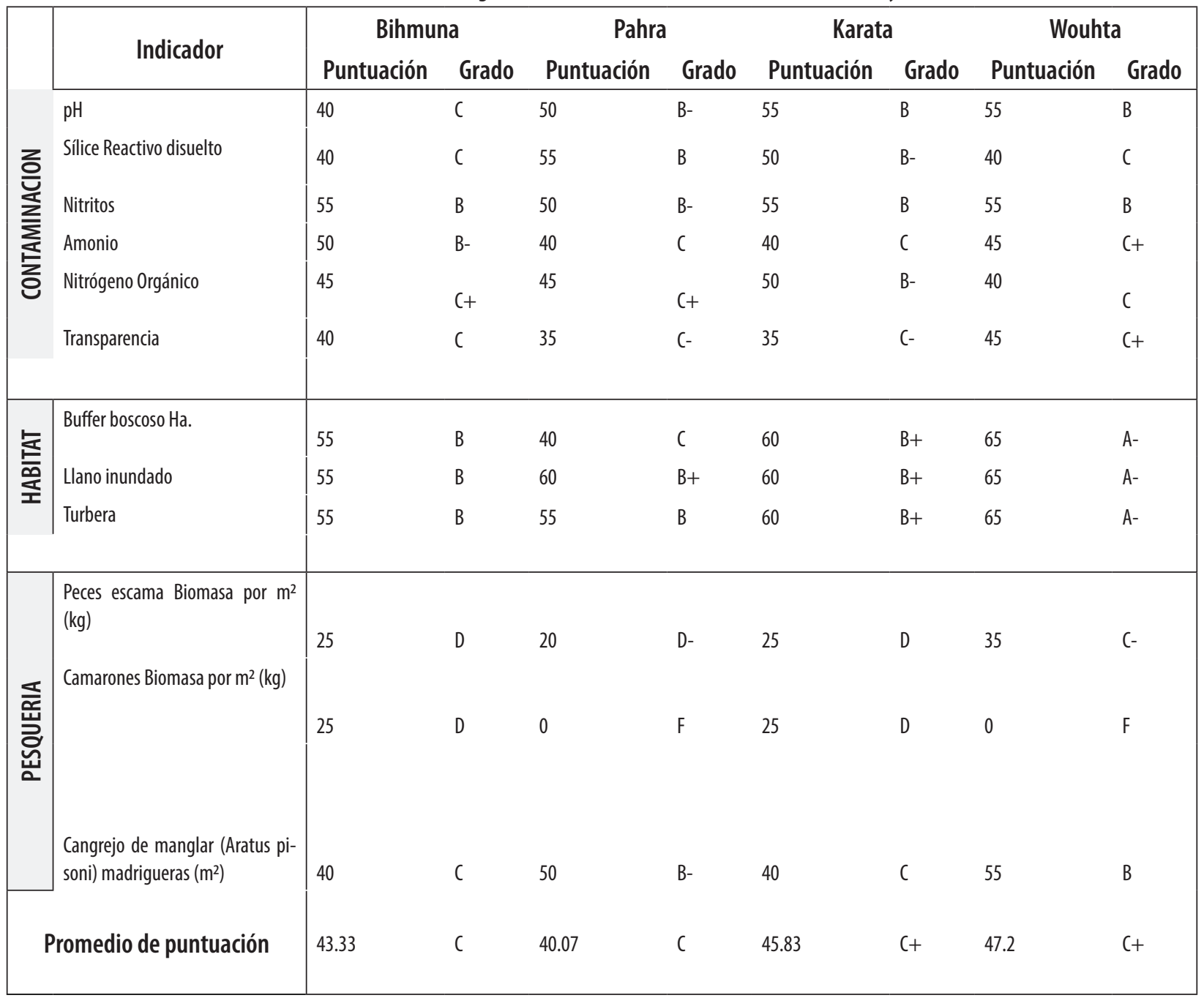

\section{Contaminación}

El agua en las lagunas naturales del Caribe Norte de Nicaragua se encuentra en buen estado ambiental, de acuerdo al análisis físico-químico realizado. Los valores de elementos nutrientes que son necesarios para la vida de plantas, peces y organismos microscópicos que conforman el fitoplancton y zooplancton, se encuentran en los rangos permisibles que no constituyen amenazas a la vida acuática.

El PH muestra un ambiente alcalino con valores entre 7.64 y 8.28, con un promedio de 7.93. La escala de medición del $\mathrm{pH}$ va de o al 14, y 7 o menos representan un ambiente ácido y por encima de 7 indica un ambiente alcalino. El pH es un factor muy importante en los sistemas químicos y biológicos de las aguas naturales. El valor del $\mathrm{pH}$ compatible con la vida piscícola está comprendido entre 5 y 9. Un pH bajo puede matar a los peses al estresar los sistemas de los animales y causarles daños físicos, lo cual a su vez los hace más vulnerables a las enfermedades. Johnson, W. (1980).

El silicio es un nutriente esencial para algunas células del fitoplancton como las diatomeas y las silicoflageladas. Los valor más alto de sílice reactivo disuelto se obtuvieron en las lagunas de Bihmuna y Wouhta con $7,56 \mathrm{mg}$. $\mathrm{L}^{-1}$. Esto puede ser debido al silicato disuelto llevado por escurrimientos de agua 
dulce al interior de las lagunas por precipitación. Estos valores son inferiores a lo registrado en lagunas costeras del norte de Yucatán, México de 119 mg. L ${ }^{-1}$. Herrera S. (1997).

La presencia de nitrógeno en forma de nitratos y nitritos en agua costeras suele interpretarse como indicativo de contaminación de origen agrícola y/o urbano. El nivel de nitrito no debe superar o,l mg N-No2-/L (Sturrun y Morgan, 1981), pudiendo ser los valores superiores tóxicos para la vida acuática. Los nitritos suelen considerarse como compuestos de bajas concentraciones en aguas someras puesto que tienden a oxidarse rápidamente pasando a nitratos. Se obtuvieron valores de $0,003 \mathrm{mg}$. $\mathrm{L}^{-1}$ en las lagunas de Bihmuna, Karata y Wouhta y el valor más alto de concentración de nitrito se registró en la laguna de Pahra con $0,010 \mathrm{mg}$. L ${ }^{-1}$.

El amonio es considerado habitualmente como nitrógeno procedente de la descomposición de materia orgánica y/o excreción producida por organismos. La concentración de amonio fue entre 0,008 $\mathrm{L}^{-1}$ y 0 . 023mg. $\mathrm{L}^{-1}$, siendo mayor en la laguna de Pahra con $0,23 \mathrm{~L}^{-1}$. Estos valores se encuentran muy por debajo de lo encontrado en las lagunas costeras de Murcia en Colombia donde registraron $0.29 \mathrm{mg}^{-1} \mathrm{~L}^{-1}$. Lopez et. al 2009.

Los nutrientes en las lagunas naturales son importantes para el desarrollo del fitoplancton y en estas muestras existe buen abastecimiento de ellos. La producción de peces en lagunas costeras está vinculada a la producción primaria (PP) de comunidades plantónicas y bentónicas. Las lagunas costeras juegan un importante papel como áreas de desove y crianza de poblaciones de peces y crustáceos juveniles (YañezArancibia et al, 1994). Cuadro No. 3.

\begin{tabular}{|c|c|c|c|c|c|c|c|c|}
\hline & & & adro No. 3. & is de pará & tros físico-quí & & & \\
\hline \multirow[b]{2}{*}{$\begin{array}{c}\text { Código de } \\
\text { laboratorio }\end{array}$} & \multirow[b]{2}{*}{$\begin{array}{c}\text { Identifica- } \\
\text { ción. De la } \\
\text { muestra }\end{array}$} & \multicolumn{7}{|c|}{ Variables analizadas } \\
\hline & & $\mathrm{pH}$ & $\begin{array}{c}\text { Alcalinidad } \\
\text { total }\end{array}$ & $\begin{array}{c}\text { Sílice } \\
\text { Reactivo } \\
\text { disuelto }\end{array}$ & Nitritos & Amonio & $\begin{array}{c}\text { Nitrógeno } \\
\text { total }\end{array}$ & $\begin{array}{c}\text { Nitrógeno } \\
\text { Orgánico }\end{array}$ \\
\hline \multirow[b]{2}{*}{ AN-0351 } & \multirow[b]{2}{*}{ Laguna de Pahra } & \multirow[b]{2}{*}{8,12} & $72,01 \mathrm{mg} \cdot \mathrm{L}^{-1}$ & & & & & \\
\hline & & & 1,440 meq. L $^{-1}$ & $0,95 \mathrm{mg} \cdot \mathrm{L}^{-1}$ & $0,010 \mathrm{mg} \cdot \mathrm{L}^{-1}$ & $0,023 \mathrm{mg} \cdot \mathrm{L}^{-1}$ & $0,954 \mathrm{mg} \cdot \mathrm{L}^{-1}$ & $0,231 \mathrm{mg} \cdot \mathrm{L}^{-1}$ \\
\hline \multirow{2}{*}{ AN-0352 } & \multirow{2}{*}{$\begin{array}{l}\text { Laguna de } \\
\text { Bihmuna }\end{array}$} & \multirow{2}{*}{8,28} & $112,01 \mathrm{mg} \cdot \mathrm{L}^{-1}$ & & & & & \\
\hline & & & 2,240 meq. $\mathrm{L}^{-1}$ & $7,56 \mathrm{mg}^{-\mathrm{L}^{-1}}$ & $0,003 \mathrm{mg} \cdot \mathrm{L}^{-1}$ & $0,008 \mathrm{mg} \cdot \mathrm{L}^{-1}$ & $0,882 \mathrm{mg} \cdot \mathrm{L}^{-1}$ & $0,201 \mathrm{mg} \cdot \mathrm{L}^{-1}$ \\
\hline \multirow[b]{2}{*}{ AN-0361 } & \multirow[b]{2}{*}{ Laguna Karata } & \multirow[b]{2}{*}{7,70} & $94,01 \mathrm{mg} \cdot \mathrm{L}^{-1}$ & & & & & \\
\hline & & & 1,880 meq. L $^{-1}$ & $1,62 \mathrm{mg}^{-\mathrm{L}^{-1}}$ & $<0,003 \mathrm{mg} . \mathrm{L}^{-1}$ & $0,021 \mathrm{mg} \cdot \mathrm{L}^{-1}$ & $0,810 \mathrm{mg} \cdot \mathrm{L}^{-1}$ & $0,171 \mathrm{mg} \cdot \mathrm{L}^{-1}$ \\
\hline \multirow[b]{2}{*}{ AN0362 } & \multirow[b]{2}{*}{ Laguna Wouhta } & & $68,01 \mathrm{mg} \cdot \mathrm{L}^{-1}$ & & & & & \\
\hline & & 7,64 & 1,360 meq. $\mathrm{L}^{-1}$ & $7,56 \mathrm{mg} \cdot \mathrm{L}^{-1}$ & $<0,003 \mathrm{mg} \cdot \mathrm{L}^{-1}$ & 0,015mg.L-1 & $0,798 \mathrm{mg}^{-L^{-1}}$ & $0,390 \mathrm{mg} \cdot \mathrm{L}^{-1}$ \\
\hline
\end{tabular}

\section{Biológico}

\section{Riqueza de fitoplancton}

Numerosos aspectos de la estructura y funcionamiento de sistemas lagunar de la zona costera inmediata de la Reserva Biológica Cayos Miskitos, influyen sobre el fitoplancton presente. El sistema lagunar es somero, rodeado de bosque de mangle, no se observó presencia de plantas flotantes, que pudieran 
ejercer efectos alelopáticos, reducción de la cantidad de luz incidente o de la concentración de nutrientes en el medio. En las lagunas de Karata y Wontha encontramos pasto que consumen los manatíes y pueden ejercer un efecto positivo sobre la riqueza y abundancia del fitoplancton generando ambientes oxigenados.

Durante los muestreos realizados en las lagunas Bihmuna, Pahra, Karata y Wouhta, se logró registrar un total de 67 especies, distribuidos en 4 Divisiones, entre las que destacan por su abundancia Bacillariophyta (diatomeas) con 40 especies y Dinophyta (dinoflagelados) con 22 especies. Ambas suman un porcentaje total de $92.53 \%$.

De acuerdo a la diversidad de especies y de divisiones encontrada entre las cuatro lagunas muestreadas en Bihmuna y Pahra se registra el mayor número de especies con 33 y 32 respectivamente de tres divisiones, mientras que en Wounta se registraron 27 especies de cuatro divisiones, entre ella Chlorophyta las que son especialmente importantes en los océanos, donde se calcula que proporcionan hasta un $45 \%$ del total de la producción primaria oceánica. Meave-del Castillo et,al. (2012). Figura No. 1.

Figura No. 1. Porcentaje de divisiones y especies de fitoplanton en lagunas costeras

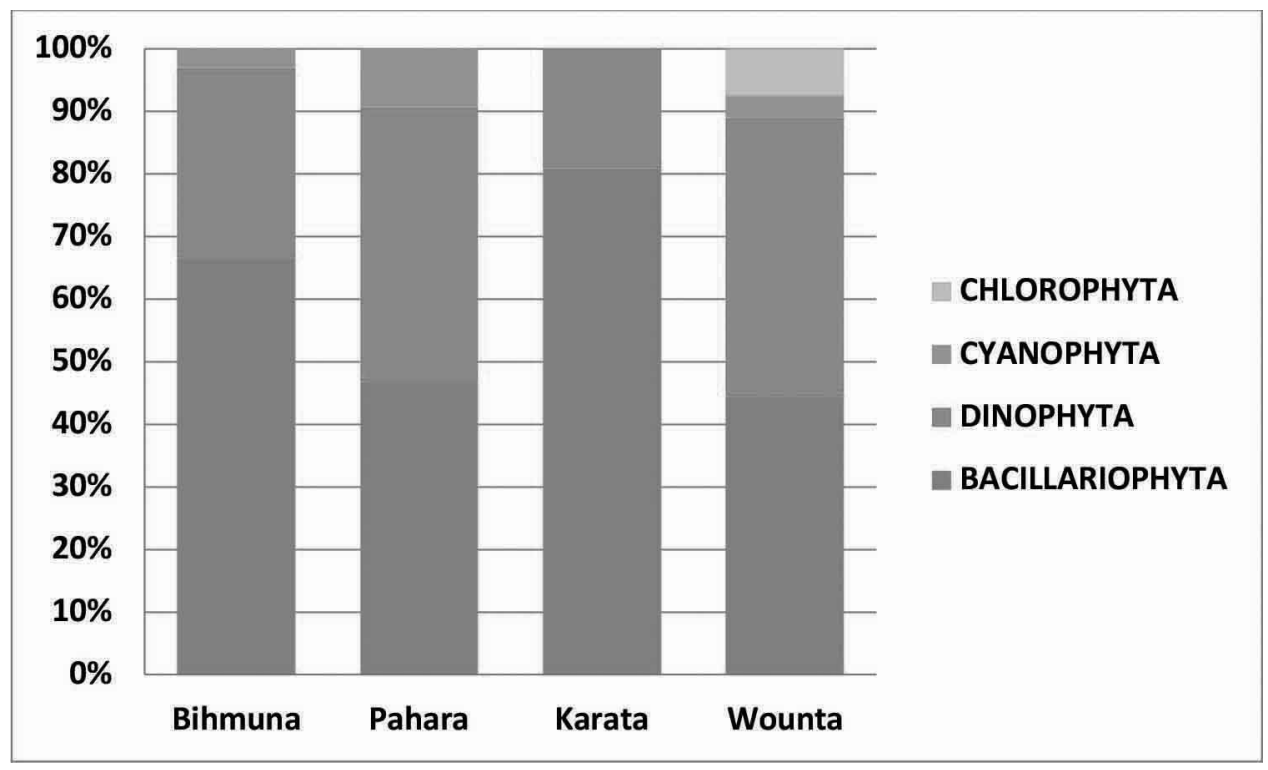

\section{Hábitat}

Las lagunas naturales del Caribe Norte de Nicaragua se encuentran rodeadas de bosque de mangle, extensas áreas de llanos inundados y llanos temporalmente inundados conocidos como turberas, que ayudan a mantener la estabilidad ecológica de la región. Este ecosistema es importante para la reproducción de organismos hidrobiológicos de valor comercial y alimenticio para la población, entre ellos camarón (penaeus sp.), Camarón de río (Macrobrachium rosenbergii) y peces. El área del espejo de agua, bosque de mangle y llanos, varía entre las lagunas, siendo mayor en la laguna de Bihmuna. Sólo el área de turberas es mayor en la laguna Karata. Cuadro No. 4.

Cuadro No. 4. Cantidad de área cubierta por los diferentes ecosistemas presentes en las lagunas naturales del Caribe Norte de Nicaragua

\begin{tabular}{|l|l|l|l|l|}
\hline \multicolumn{1}{|c|}{ Laguna } & Area del espejo de agua. & Area de manglar. Ha & Área de Llano inundado. Ha. & Área de turbera. Ha. \\
\hline Bihmuna & $147.54 \mathrm{~km}^{2}$ & $4,253.19$ & 908.83 & 852.35 \\
\hline Pahra & $101.30 \mathrm{~km}^{2}$ & $3,160.22$ & 1002.95 & 532.49 \\
\hline Karata & $38.82 \mathrm{~km}^{2}$ & $2,307.97$ & 1078.8 & 1120.46 \\
\hline Wouhnta & $88.52 \mathrm{~km}^{2}$ & $4,068.02$ & 4011.89 & 2436.7 \\
\hline
\end{tabular}


El bosque de manglar en las lagunas costeras del Caribe de Nicaragua es muy compacto, aunque ha sido fuertemente azotado por los fenómenos naturales. La cobertura del manglar en estas lagunas está compuesta por varias especies diferentes, las cuales se encuentran formando rodales puros o asociaciones mixtas con otras especies de mangle.

Las especies de mangle que se lograron identificar durante los muestreos fueros cuatro: Mangle rojo (Rhizophora mangle) el que tiene mayor dominancia, seguido de Mangle negro (Avicennia germinans), Mangle blanco (Laguncularia racemosa) y Mangle hembra (Pelliciera rhizophorae). En la laguna Wouhnta la altura promedio y el DAP de los árboles resultó mayor. Cuadro No. 5.

Cuadro No. 5 Promedio de mediciones dasométricas de los árboles de mangle en las lagunas naturales del Caribe Norte de Nicaragua

\begin{tabular}{|l|l|l|l|l|}
\hline \multicolumn{1}{|c|}{ Laguna } & Área de mangle Ha. & \multicolumn{1}{c|}{ Altura media Mts. } & DAP promedio & \multicolumn{1}{c|}{ Regeneración Natural Planta m $^{2}$} \\
\hline Bihmona & $4,253.19$ & 15,25 & 18,21 & 17 \\
\hline Pahara & $3,160.22$ & 13,3 & 11,7 & 7 \\
\hline Karata & $2,307.97$ & 23,5 & 42 & 3 \\
\hline Wouhnta & $4,068.02$ & 17,86 & 32,17 & 13 \\
\hline
\end{tabular}

La distribución diamétrica de los árboles de mangle en las cuatro lagunas muestreadas presenta un patrón diferente, en Bihmuna presenta una forma de J invertida, en Pahra el patrón es inusual, con menos árboles de menor diámetro y a medida que el diámetro se hace mayor crese el número de árboles. En Karata, presenta mayor número de árboles jóvenes, luego entre 10 y 15 centímetros de diámetro no presenta árboles y luego aparece con diámetro superior a 15 centímetro. En Wouhnta el patrón es diferente menos árboles entre 10 y $19.9 \mathrm{~cm}$. De diámetro, aumentando el número de árboles con diámetro superior a $20 \mathrm{~cm}$. Esta característica podría deberse a que son bosques maduros cerrados y los árboles de mayor diámetro son los que predominan. Figura No. 2.
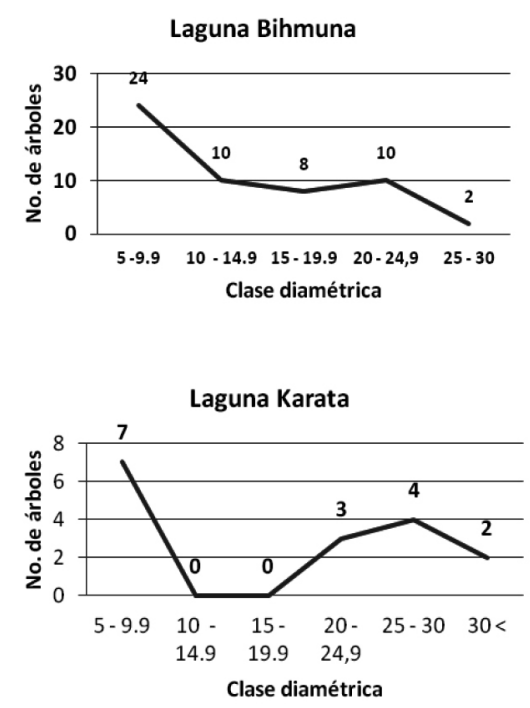

Laguna Pahara

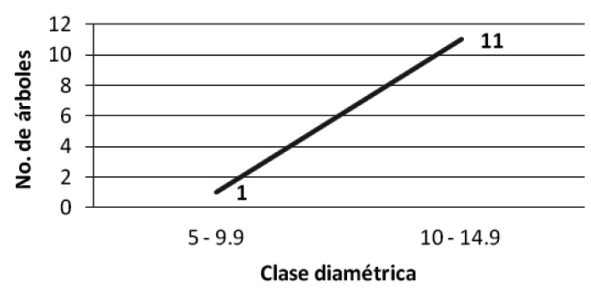

Laguna Wouhnta

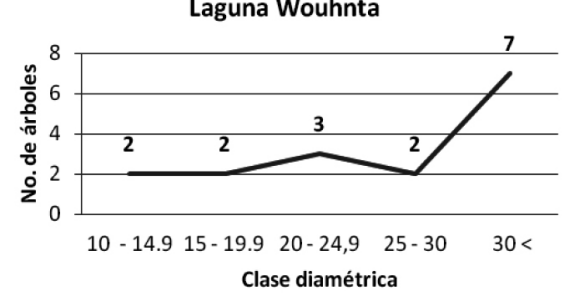

Figura No. 2. Representación gráfica de distribución diamétrica de árboles de mangle en lagunas continentales.

\section{Llanos inundados}

Terreno llano o un poco ondulado adyacente a ríos o arroyos que experimentan inundaciones. Llanuras aluviales que tuvieron su génesis en el depósito de materiales arrastrados por ríos o arroyos en situaciones 
de caudales extremos. Los materiales transportados al depositarse han creado, a lo largo del tiempo planicies poco inclinadas con suelos fértiles muy propicios para la agricultura. Estos terrenos inundables son, en ocasiones, ecosistemas muy ricos y frágiles. La vegetación predominante es este sistema es la arbustiva (Melastomaceae, Cyperaceae y otras), el uso que la población indígena da a esta zona es para la caza, en pequeña escala el aprovechamiento especies forestales menores para leña y pastoreo de ganado vacuno. Existen en esta zona igualmente planicies inundadas dominadas por pinos (Pinus caribaea) límite sur de este tipo de ecosistemas inundables en el hemisferio Norte de América. BICU - CIUM 2000.

\section{Turberas}

Estos ecosistemas singulares se forman por la acumulación de los tejidos de plantas que crecen sobre los restos de otras ya descompuestas. Forman así una serie de estratos de tejidos vegetales muertos que se acumulan con el paso del tiempo formando el material que llamamos turba, el cual puede alcanzar varios metros de espesor al cabo de miles de años. Normalmente, las turberas se forman sobre suelos de tipo silíceo, es decir, ácidos y pobres en nutrientes. La materia vegetal transformada en turba es, básicamente, materia orgánica. Por ello, las turberas son grandes sumideros de carbono. Se calcula que una hectárea de turbera puede almacenar entre 3 y 3,5 veces más carbono que una hectárea de bosque tropical. Para cumplir con el Protocolo de Kioto de cara a reducir la emisión de gases de efecto invernadero y evitar el calentamiento global del planeta, es fundamental también la conservación de estos sumideros de carbono como almacén de este elemento, evitando su salida a la atmósfera (Fundación Santander, 2006).

\section{Biomasa en lagunas naturales}

En cuanto a la presencia del cangrejo de manglar (Aratus pisoni), fue muy variada, pero superior en la laguna de Gouhnta 15 madrigueras $\mathrm{m}^{2}$, seguido de la laguna de Pahra con 10 madrigueras $\mathrm{m}^{2}$ en Bismuna 6 madrigueras $\mathrm{m}^{2}$, Karata 6 madrigueras $\mathrm{m}^{2}$.

La pesca en su mayoría fueron camarones, sardina,; asimismo, capturaron palometa, robalo y mojarra, en estado juvenil. En todos los sitios alta población de medusas. En los muestreos la pesca de escama resultó baja, aunque en todas existen datos la biomasa calculada por metro cuadrado fue mayor en la laguna de Wouhta con 19,62 kg. La mayor cantidad de biomasa se obtuvo de medusas. Los resultados de la biomasa de para cada laguna muestreada se presentan en el Cuadro No. 6.

Cuadro No. 6. Biomasa de peces y camarones obtenida en las lagunas naturales del Caribe de Nicaragua

\begin{tabular}{|l|l|l|l|l|l|}
\hline \multirow{2}{*}{ Laguna } & \multirow{2}{*}{ Área de muestreo $\left(\mathbf{m}^{2}\right)$} & \multicolumn{2}{|c|}{ Biomasa de Peces } & \multicolumn{2}{c|}{ Biomasa de Camarones } \\
\cline { 3 - 6 } & & Peso Total $(\mathbf{g})$ & Biomasa por $\mathbf{m}^{2} \mathbf{( g )}$ & Peso Total $(\mathbf{g})$ & Biomasa por $\mathbf{~ m}^{2} \mathbf{( g )}$ \\
\hline Bihmuna & 718.5 & 6556 & 9.12 & 3095 & 4.3 \\
\hline Pahra & 574.8 & 1793 & 3.11 & 0 & 0 \\
\hline Karata & 479 & 5344 & 11.15 & 965 & 2.02 \\
\hline Wouhnta & 814.3 & 15977 & 19.62 & 0 & 0 \\
\hline
\end{tabular}

\section{Conclusiones}

Las lagunas naturales presentes en la Reserva Biológica Cayos Miskitos, se encuentran en buen estado de conservación. La interacción de los ecosistemas lagunar, el manglar, los llanos inundados y el Mar Caribe le confieren una serie de atributos que las hacen mantener altos valores de biodiversidad, de conservación y de paisajes que las convierten en sitios especiales que deben seguir conservados para garantizar que los pobladores continúen haciendo uso de los recursos naturales como lo han realizado a través de la historia. 
El humedal presente en las lagunas naturales de Cayos Miskitos, se mantienen en buen estado de conservación. El índice de salud de las lagunas indica que no existe mayor perturbación y continúan realizando su función ecológica de protección, conservación y desarrollo de la biodiversidad.

Las áreas de mangle demuestran su función en la protección de la costa y las comunidades indígenas. Solo el sector más próximo al mar presenta afectación por los fenómenos naturales, pero muestran recuperación. Los cangrejos del mangle (Aratus pisoni), se encuentran bien representados en las cuatro lagunas y son un indicativo de buen estado de salud del manglar.

Los comunitarios hacen uso sostenible de las lagunas naturales tal y como lo han realizado durante todo el tiempo.

\section{Agradecimiento}

Esta publicación obtuvo el financiamiento del Proyecto Ecosistemas Costeros, auspiciado por: Unión Europea, Dka Austria, Cooperación Austriaca para el Desarrollo y Horizontzooo.

\section{Lista de referencias}

Alzieu, C. (1994). El agua medio de cultivo. G. Barnabé, (Ed.). Acuacultura (I). Ediciones Omega S. A. España. p. 1-27.

BICU - CIUM (2000). Ficha informativa de los humedales de RAMSAR. Cayos Miskitos y franja costera inmediata.

Contreras, F. y O. Castañeda (2004). La biodiversidad de las lagunas costeras. Ciencias 76: 46-56.

Herrera Silveira, J. A., (1997). Biodiversidad de productores primarios de lagunas costeras del norte de Yucatán, México. Instituto Politécnico Nacional. Centro de Investigación y de Estudios Avanzados-Mérida Informe final SNIB-CONABIO proyecto No. Bo19. México D. F.

Johnson, W. W. (1980). Handbook of acute toxicity of chemicals to fish and aquatic invertebrales. Fish and Wildlife, Service. Resource Publication 137. United States Departernent of the Interior, Washington D.C.

Kjerfve, B. (1994). Coastal Lagoon Processes. Elsevier.New York 577 p.

López M, Pulido G, Serrano A, Gaytan J, Monks Wy López M. (2012). Evaluación estacional de las variables físicoquímicas del agua de la Laguna de Tampamachoco, Veracruz, México.

López L, Andrés Paredes Giménez, Nuria Alcaraz Oliver, Javier Gilabert (2009). Análisis de parámetros físicos, químicos y biológicos en las aguas costeras de la región de Murcia. Cartagena, Colombia.

Santander (2006). Manuales de desarrollo sostenible. Conservación y restauración de turberas.

Stumm W. and J.J. Morgan (1981). Aquatic Chemistry. Nueva York.

Save de Bay (2016). Estado de la Bahía Chesapeake. Informe técnico.

Tabilo -. Valdivieso E. (1999). El beneficio de los Humedales en América Central. El potencial de los humedales para el desarrollo. WWF.

Yañez-Arancibia A, AL Lara-Dominguez \& D Pauly (1994). Coastal lagoons as fish habitats. In: Kjerfve B (ed). Coastal lagoon processes pp. 363-376. Elsevier, Amsterdam. 